\title{
Diffusion Weighted Imaging in Acute Attacks of Multiple Sclerosis
}

\author{
Yasmin Davoudi, ${ }^{1}$ Mohsen Foroughipour, ${ }^{2}$ Reza Torabi, ${ }^{3}$ Parvaneh Layegh, ${ }^{1}$ Nassim Matin, ${ }^{4}$ and Ali \\ Shoeibi ${ }^{2,}$ \\ ${ }^{1}$ Department of Radiology, Imam Reza Hospital, School of Medicine, Mashhad University of Medical Sciences, Mashhad, Iran \\ ${ }^{2}$ Department of Neurology, Ghaem Hospital, School of Medicine, Mashhad University of Medical Sciences, Mashhad, Iran \\ ${ }^{3}$ Department of Radiology, School of Medicine, Mashhad University of Medical Sciences, Mashhad, Iran \\ ${ }^{4}$ School of Medicine, Tehran University of Medical Sciences, Tehran, Iran \\ "Corresponding author: Ali Shoeibi, Department of Neurology, Ghaem Hospital, School of Medicine, Mashhad University of Medical Sciences, P. O. Box: 9197969764, Mashhad, \\ Iran. Tel: +98-9155137344, Fax: +98-5138429828, E-mail: shoeibia@mums.ac.ir
}

Received 2014 July 05; Revised 2014 October 04; Accepted 2014 October 07.

\begin{abstract}
Background: Multiple sclerosis (MS) is one of the most common autoimmune disorders of the central nervous system. In spite of various imaging modalities, the definitive diagnosis of MS remains challenging.

Objectives: This study was designed to evaluate the usefulness of diffusion weighted imaging (DWI) in the diagnosis of acute MS attack and to compare its results with contrast enhanced MRI (CE-MRI).

Patients and Methods: In this cross sectional study, seventy patients with definite diagnosis of relapsing-remitting MS were included. CE-MRI using $0.1 \mathrm{mmol} / \mathrm{kg}$ gadolinium as well as DWI sequences were performed for all patients. The percentage of patients with positive DWI was compared with the results of CE-MRI and the consistency between the two imaging modalities was evaluated. Moreover, the relationship between the time of onset of patient's symptoms and test results for both methods were investigated. Results: CE-MRI yielded positive results for $61(87 \%)$ patients and DWI yielded positive for 53 (76\%) patients. In fifty patients ( $71.42 \%)$, both tests were positive and in six cases (8.57\%), both were negative. The test results of three patients turned out to be positive in DWI, while they tested negative in CE-MRI. There was no significant relationship between the results of CE-MRI as well as DWI and the time of imaging from the onset of symptoms.

Conclusion: These data indicate that while CE-MRI will depict more positive results, there are cases in which DWI will show a positive result while CE-MRI is negative. We suggest that the combination of these two imaging modalities might yield more positive results in diagnosing acute MS attack giving rise to a more accurate diagnosis.
\end{abstract}

Keywords: Diffusion Magnetic Resonance Imaging, Multiple Sclerosis, Magnetic Resonance Imaging

\section{Background}

Multiple sclerosis (MS), the most common autoimmune neurological disorder, affects about 2.5 million people around the world (1). Iran is one of the geographic areas in the world that seems to have a high prevalence of this disease (2). The disease pathology consists of multifocal demyelination and to less extent, axonal injury in the central nervous system due to autoimmune inflammatory processes (3). Despite various imaging modalities, the definite diagnosis of the disease is still challenging. However, development of different magnetic resonance imaging (MRI) techniques has revolutionized the diagnosis and has led to early and more precise diagnosis. MRI is now considered as the most sensitive imaging technique for identifying MS lesions $(1,4,5)$ and has improved treatment and therefore, prognosis of patients with MS because of the possibility of earlier diagnosis, which is critical in the treatment of the disease.

Myelin breakdown and formation of white matter le- sions is associated with disruption in the blood-brain barrier as the primary trigger of tissue inflammation and edema. Perivascular inflammation, demyelination, gliosis, and axonal injury are the main pathologic features of MS plaques (1). The edema results in different signal intensities in different sequences of MRI as well as contrast enhancement (CE) in CE-MRI. Conventional MRI techniques such as T1-weighted without contrast, T2-weighted, and T1weighted imaging with gadolinium enhancement are traditional MRI techniques employed in the diagnosis and follow-up of MS patients $(6,7)$. The development of quantitative (non-conventional) MR techniques opened a new era in understanding the histopathology of MS. Since the primary inflammatory changes in MS consist of the cytotoxic type of edema followed by vasogenic edema, it may induce alterations in apparent diffusion coefficient (ADC), an index used in DWI. In fact, diffusion weighted imaging (DWI) has shown alterations in the white matter in acute MS patients $(8,9)$. 
Since the study of Larsson et al. (10), who introduced DWI as a promising method in identifying acute MS lesions in 1993, the potential role of DWI and its diagnostic capability has been a controversial subject. (10-12) There are a few studies worldwide that have discussed DWI as a diagnostic imaging method with a reported capability comparable to conventional CE-MRI. $(9,13)$ Nevertheless these studies have revealed conflicting results.

\section{Objectives}

This compelled us to design a study with the aim of evaluating the consistency between the two imaging modalities and to evaluate the probable role of DWI in the diagnosis of acute MS attacks.

\section{Patients and Methods}

\subsection{Participants}

In this cross sectional study, we examined seventy patients with the definite diagnosis of relapsing-remitting MS who were referred to the neurology department of our teaching hospital with an acute MS attack. Diagnosis of definite MS was based on 2010 McDonald criteria (14) and an acute attack was defined as the presence of new objective neurological signs lasting for at least 24 hours and were compatible with an MS attack. Diagnosis of an acute MS attack was made by two expert neurologists (M. F \& A. $\mathrm{Sh}$ ) based on clinical findings. All the cases were receiving disease-modifying treatment. Those who had fever, history of neurosurgical operation, and those who had used corticosteroids or immunosuppressant agents during the last month prior to their visit were excluded. In order to establish whether the interval between symptom onset and performing MRI had any impact on the diagnostic capability of MRI, we categorized patients into three groups: cases whose MRI was performed 1 - 4 days, 5 - 9 days and 10 - 14 days after the onset of their new symptoms.

\subsection{Test Methods}

All patients underwent a brain MRI employing a 1.5 Tesla Machine (Siemens Symphony). CE-MRI using 0.1 $\mathrm{mmol} / \mathrm{kg}$ gadolinium as well as DWI sequences were performed for all patients. CE-MRI was performed $10 \mathrm{~min}$ utes after gadolinium injection (DOTAREM $0.5 \mathrm{mmol} / \mathrm{ml}$, France) using a T1W image (TR: 400 - 500, TE: 8, slice thickness: $5 \mathrm{~mm}$ ). Diffusion weighted images was performed in b value 1: 0 , b value 2: 500 , b value 3: 1000 . Noise level: 40, band width: $952 \mathrm{~Hz} / \mathrm{px}$, echo spacing: $1.13 \mathrm{~ms}$, TR: 3300 3500, TE: 94 - 118).
Two radiologists (Y.D \& P. L) evaluated all the images together and by consensus. Furthermore, prior to the study, they had calibrated with each other in terms of diagnosing positive plaques. We included only the cases in which both reviewers had the same opinion about imaging findings. They were both experienced radiology consultants in our teaching hospital. Radiologists were blinded to time duration between attacks and imaging recording as well as whether it is a new or old MRI. They were also blinded to the results of DWI while interpreting CE-MRI images and vice versa. A positive CE-MRI was considered whenever a hypointense TiW lesion showed increased signal intensity in T1W image taken 10 minutes after gadolinium injection. A positive DWI result was defined as any lesion compatible with a true plaque in traditional sequences of MRI with increased signal intensity in DWI that showed restricted diffusion in ADC mapping. Detection of even one positive lesion was regarded as a positive result both in DWI and CEMRI.

\subsection{Ethics}

The regional ethical committee of Mashhad University of Medical Sciences approved the study. All patients signed informed consent forms.

\subsection{Statistical Methods}

The results of the two imaging modalities were compared. Chi square and Fisher's exact tests were used to analyze qualitative data. McNemar test was used to assess the difference of distribution of positive results between the two methods. SPSS software for Windows (version 12.0, SPSS, Inc., Chicago, IL, USA) was employed for data analysis. $P$ values less than 0.05 were considered statistically significant.

\section{Results}

\subsection{Participants}

Among the cases, 49 (70\%) were female and $21(30 \%)$ were male. The mean age of our patients was $32 \pm 7.3$ years (18-53). From the 70 referred patients, 23 cases (33\%) were studied between the first and fourth day of the onset of the attack, 32 patients (46\%) were studied during days 5 to 9 , and 15 patients (21\%) were studied from days 10 to 14 .

\subsection{Test Results}

Sixty-one patients (87\%) showed contrast enhancement in MRI, and 53 patients (76\%) showed restricted diffusion in DWI. There was no statistically significant relationship between the time of onset of symptoms and positive findings in either CE-MRI $(\mathrm{P}=0.897)$ or DWI $(\mathrm{P}=0.528)$ (Tables 1 and 2). In both methods, MRI abnormalities were not significantly related to the age of the patients. 
Table 1. CE-MRI Findings Based on the Day of Performing MRI ${ }^{\mathrm{a}, \mathrm{b}}$

\begin{tabular}{|c|c|c|c|c|}
\hline \multirow{2}{*}{ CE-MRI } & \multicolumn{4}{|c|}{ Timing } \\
\hline & 1st to 4th Day & 5th to 9th Day & 10th to 14th Day & Total \\
\hline Positive & $21(30)$ & $27(38.6)$ & $13(18.6)$ & $61(87.1)$ \\
\hline Negative & $2(2.9)$ & $5(7.1)$ & $2(2.9)$ & $9(12.9)$ \\
\hline Total & $23(32.9)$ & $32(45.7)$ & $15(21.4)$ & $70(100)$ \\
\hline \multicolumn{5}{|c|}{$\begin{array}{l}\text { Abbreviation: CE-MRI, contrast enhanced magnetic resonance imaging } \\
{ }^{\mathrm{a}} \text { Exact test } \mathrm{P} \text { value }=0.897 \\
{ }^{\mathrm{b}} \text { Values are Presented No. (\%) }\end{array}$} \\
\hline
\end{tabular}

Table 2. The Results of DWI Based on the Day of Performing MRI ${ }^{\mathrm{a}, \mathrm{b}}$

\begin{tabular}{lccc}
\hline \multirow{2}{*}{ DWI } & \multicolumn{2}{c}{ Timing } \\
\cline { 2 - 4 } & 1st to 4th day & 5th to 9th day & 10th to 14th day \\
\hline Positive & $17(24.3)$ & $27(38.6)$ & $9(12.9)$ \\
Negative & $6(8.6)$ & $5(7.1)$ & $6(8.6)$ \\
Total & $23(32.9)$ & $32(45.7)$ & $17(25)$ \\
\hline
\end{tabular}

Abbreviation: DWI, diffusion weighted imaging

${ }^{\mathrm{a}}$ Chi square $\mathrm{P}$ value $=0.528$

${ }^{\mathrm{b}}$ Values are presented No. (\%)

\subsection{Estimates}

CE-MRI showed enhanced MS lesions in $87 \%$ (61) of the patients. A restricted diffusion was detected in $76 \%$ (53) of the patients. Of these 53 patients, three showed no enhancing lesion in their CE-MRI. In fifty patients (71\%), both tests were positive and in six cases ( $8 \%$ ), both were negative (Table 3). The percent of patients with positive CE-MRI were not significantly different from those with positive DWI (P $=0.5)$. Using McNemar test, there was a difference between these two methods in positive results (i.e., CE shows more lesions $)(\mathrm{P}=0.057)$.

Table 3. Cross Tabulation of the Results in CE-MRI and DWI

\begin{tabular}{lcc}
\hline \multirow{2}{*}{ DWI } & \multicolumn{2}{c}{ CE-MRI } \\
\cline { 2 - 3 } & Positive & Negative \\
\hline Positive & $50(71.4)$ & $3(4.3)$ \\
Negative & $11(15.7)$ & $6(8.6)$ \\
\hline
\end{tabular}

Abbreviations: CE-MRI, contrast enhanced magnetic resonance imaging; DWI, diffusion weighted imaging

${ }^{\mathrm{a}}$ Chi square $\mathrm{P}$ value $=0.528$

\section{Discussion}

The main interest of this study was to evaluate the consistency of CE-MRI and DWI in diagnosing acute MS attack.
This study showed a borderline P value in McNemar test. It means that CE-MRI could probably detect more lesions than DWI. Although the definite remark needs power analysis, it should be mentioned that even the borderline $\mathrm{P}$ value is very considerable and will lead to the conclusion that CE-MRI is more efficient than DWI, but this efficiency is not so considerable. Similarly, in 2014, Lo et al. studied 22 patients with acute MS attacks (384 plaques) and found significant correlation between contrast enhancement in CEMRI and restricted diffusion in DWI. They concluded that although CE-MRI cannot be replaced by DWI for demonstration of dissemination in time which is necessary in MS diagnosis, DWI can be used as a screening tool when performing CE-MRI is a concern for the patient (13). One of the most striking findings in our study was that three of our patients turned out to have restricted diffusion in DWI sequences, while their CE-MRI became negative. Since none of our patients had risk factors of ischemic brain lesions, and the location and characteristics of the resulting images were quite compatible with an MS plaque, it cannot be argued that these three lesions might have been false positive results. Using both methods, positive cases increased from $87.1 \%$ to $91.4 \%$ (totally 64 positive case; 3 additional cases besides 61 positive cases of CE-MRI). These results support the assumption of some researchers who believe that these two modalities should be used in combination, although each method can individually show the lesions in 
$80-90 \%$ of the patients (13). It seems that the final decision about using DWI in combination with CE-MRI is a matter of clinical importance and needs cost-benefit consideration by the clinician.

DWI is basically the attenuation of signal density based on the random Brownian motion of water molecules influenced by a magnetic field gradient. The diffusion of water molecules is controlled by the cell membrane components and is not "free". Water molecule mobility is restricted within the myelin. When there is myelin breakdown, the pattern of water diffusion is altered due to the modified structural barrier and broken integrity and this could be shown by an increased apparent diffusion coefficient (ADC) in DWI (12). Therefore, the finding of patients being positive for DWI could not have been due to a false positive finding. These three patients revealed negative test results when subjected to the CE-MRI technique. CEMRI is considered as the imaging modality of choice in MS patients. However, it should be noted that the exact optimal timing of image acquisition in CE-MRI has been a matter of dispute. While some investigators believe that the optimum image acquisition time is 5 minutes after the injection of gadolinium (Gd), others have reported that a 10minute interval is more sufficient (15). It might be that the exact optimum timing is yet to be determined. In the lack of an unequivocally accepted protocol, we used a 10minute interval after the injection of gadolinium and after we took the CE-MRI images. This might have resulted missing of three cases in CE-MRI.

Increased ADC was first reported as one of the characteristics of MS lesions in DWI, yet nonspecific and not helpful in distinguishing lesions of acute MS attacks. (11, 16) However, after longitudinal and case studies, it was revealed that in acute attacks of MS, the cytotoxic edema causes a reduction in ADC in the acute phase, which is then converted to a normal or increased signal along with the inflammatory vasogenic edematous changes in the subsequent days (11, 17-20). Using diffusion weighted MR imaging was first proposed by Larsson et al. (10). They evaluated 25 people and measured the water diffusion. They found that water diffusion is higher in MS plaques. Besides, they showed that it is higher in acute plaques in comparison with chronic ones. They concluded that the increased diffusion might be due to an increase in the extracellular water space caused by demyelination (10). In 1996, Iwasawa et al. (21) studied the characteristics of contrast enhanced MRI and DWI of the optic nerve in eight patients with MS. Four cases of acute optic neuritis, nine cases with chronic neuritis and seven normal volunteers were evaluated. Authors found a significantly higher ADC in chronic optic neuritis compared to the normal nerves. Most patients with acute neuritis showed re- stricted diffusion (21). Another MS case reported by Bhatia et al. (22) illustrated restricted diffusion of a lesion in the right centrum semiovale with low ADC, along with multiple juxta-cortical round and ovoid hyperintense lesions in FLAIR without contrast enhancement in a young woman with symptoms of weakness and numbness in the left side of the body. The lesion with low ADC was responsible for the symptoms, which was not detected in the other modalities (22). In a study by Balasubramanya et al. (23) in 2006, eight patients with acute disseminated encephalomyelitis (ADEM) were evaluated using conventional MRI techniques, MR spectroscopy and DWI. Of these, three patients were imaged during the first seven days (acute) and the others were evaluated after seven days (subacute stage). Acute lesions demonstrated restricted diffusion, while subacute lesions had free diffusion and a reduction in NAA/Cho. They suggested that this finding might help staging the disease (23). Yurtsever et al. (9) studied 50 patients with acute MS attacks and 18 healthy controls and showed that ADC value of active plaques is significantly higher than normal appearing white matter of both MS patients and healthy population. They also showed that the ADC value of normal appearing white matter in MS patients is significantly different from those of healthy controls. They concluded that the white matter of MS patients would show signal abnormalities even in the early stages of the disease if the patient were investigated precisely (9).

It has been reported that MS lesions do not always show the same signal attenuation over time (19, 24-26). We did not find any significant association between the times elapsed from the onset of the symptoms and imaging acquisition and the number of positive cases (Tables 1 and 2). One of the reasons for such an apparent inconsistency between the results of our study and others might be that other studies evaluated different types of MS, while our study was focused on only one type of the disease-relapsing remitting MS (RRMS). More investigations are warranted to clarify distinctions between different types of MS based on the different imaging findings over time. By investigating different presentations of various methods of MRI in different types of MS, we might be able to classify the type of disease based on the first MRI rather than clinical symptoms. This, in fact, might result in sooner initiation of the appropriate treatment. However, the cons and pros should be precisely studied, with regards to both the costs and the adverse effects. If we can reach higher sensitivities by combining DWI with other advanced MRI techniques, the imaging modality of choice might be changed, leading to earlier diagnosis and more cost effective treatments.

One of the shortcomings of our study was that we performed our analysis at a "patient level" rather than "lesion level". Nevertheless, we believe that even the analysis 
at "patient level" rather than "lesion level" is of value and even clinically more relevant. As a whole, we are encountered with a patient rather than the lesion. The presence of three patients with negative CE-MRI and positive DWI results indicates that at least in three patients no single enhanced plaque was detected in MRI whilst at least one active plaque was detected by DWI in those patients. The other limitation of our study was that we encountered the DWI images subjectively. Maybe, if the actual ADC values were calculated, the results would be more objective.

This study showed that DWI might be able to pick up lesions in some patients in whom there were no detectable lesions when CE-MRI was used. To our knowledge, reports that corroborated with this aspect of our findings are few and limited to case reports. As such, our study should be regarded as a pilot study. We recommend more studies with a greater number of patients and considering their clinical findings in order to come up with a more powerful conclusion. The essence of our findings was that although CEMRI is more sensitive than DWI in depicting active plaques, there are occasions in which CE-MRI may fail to show active lesions that were detected by DWI.

We strongly recommend further future investigations based on the plaque as a unit of analysis rather than the patients, along with evaluation of the possible relationship between the location of the plaques and alteration of DWI. This will further elucidate the diagnostic reliability of DWI as compared to CE-MRI.

The findings of this study support the idea that combination of DWI with other traditional MRI techniques for the diagnosis of acute MS attack might be beneficial in terms of detecting a greater number of positive patients. More investigations using greater number of patients are warranted.

\section{Footnotes}

Authors' Contributions: Yasmin Davoudi, Mohsen Foroughipour and Parvaneh Layegh were responsible for study concept, and design. Parvaneh Layegh was responsible for acquisition of data. Analysis and interpretation of the data was carried out by Reza Torabi. Drafting of the manuscript was performed by Yasmin Davoudi and Ali Shoeibi. Critical revision of the manuscript for important intellectual content was performed by Yasmin Davoudi. Reza Torabi and Nassim Matin were responsible for statistical analysis. Administrative, technical, and material support was done by Mohsen Foroughipoor, and finally, study supervision was carried out by Yasmin Davoudi and Mohsen Foroughipour.

Funding/Support: This research was funded by Mashhad University of Medical Sciences and the patients did not pay for their imaging. This study received a research grant from Mashhad University of Medical Sciences.

\section{References}

1. Hygino da Cruz LJ, Batista RR, Domingues RC, Barkhof F. Diffusion magnetic resonance imaging in multiple sclerosis. Neuroimaging Clin N Am. 2011;21(1):71-88. doi: 10.1016/j.nic.2011.02.006. [PubMed: 21477752].

2. Izadi S, Nikseresht A, Sharifian M, Sahraian MA, Hamidian Jahromi A, Aghighi M, et al. Significant increase in the prevalence of multiple sclerosis in iran in 2011. Iran J Med Sci. 2014;39(2):152-3. [PubMed: 24644387].

3. Dutta R, Trapp BD. Relapsing and progressive forms of multiple sclerosis: insights from pathology. Curr Opin Neurol. 2014;27(3):271-8. doi: 10.1097/WCO.0000000000000094. [PubMed: 24722325].

4. Traboulsee AL, Li DK. The role of MRI in the diagnosis of multiple sclerosis. Adv Neurol. 2006;98:125-46. [PubMed: 16400831].

5. Rahimian N, Saligheh Rad H, Firouznia K, Ebrahimzadeh SA, Meysamie A, Vafaiean $\mathrm{H}$, et al. Magnetic resonance spectroscopic findings of chronic lesions in two subtypes of multiple sclerosis: primary progressive versus relapsing remitting. Iran J Radiol. 2013;10(3):128-32. doi: 10.5812/iranjradiol.11336. [PubMed: 24348597].

6. Simon JH, Bermel RA, Rudick RA. Simple MRI metrics contribute to optimal care of the patient with multiple sclerosis. AJNR Am J Neuroradiol. 2014;35(5):831-2. doi:10.3174/ajnr.A3937. [PubMed: 24699092].

7. Alizadeh A, Roudbari SA, Heidarzadeh A, Kouhsari M. Comparison between immediate and delayed imaging after gadolinium chelate injection for detecting enhanced lesions in multiple sclerosis. Iran J Radiol. 2010;7(4):235-9.

8. Phuttharak W, Galassi W, Laopaiboon V, Laopaiboon M, Hesselink JR. Abnormal diffusivity of normal appearing brain tissue in multiple sclerosis: a diffusion-weighted MR imaging study. J Med Assoc Thai. 2007;90(12):2689-94. [PubMed: 18386722].

9. Yurtsever I, Hakyemez B, Taskapilioglu O, Erdogan C, Turan OF, Parlak M. The contribution of diffusion-weighted MR imaging in multiple sclerosis during acute attack. Eur J Radiol. 2008;65(3):421-6. doi: 10.1016/j.ejrad.2007.05.002. [PubMed: 17587524].

10. Larsson HB, Thomsen C, Frederiksen J, Stubgaard M, Henriksen O. In vivo magnetic resonance diffusion measurement in the brain of patients with multiple sclerosis. Magn Reson Imaging. 1992;10(1):7-12. [PubMed: 1545684].

11. Castriota-Scanderbeg A, Sabatini U, Fasano F, Floris R, Fraracci L, Mario MD, et al. Diffusion of water in large demyelinating lesions: a followup study. Neuroradiology. 2002;44(9):764-7. doi: 10.1007/s00234-0020806-y. [PubMed: 12221449].

12. Rueda-Lopes FC, Hygino da Cruz LJ, Doring TM, Gasparetto EL. Diffusion-weighted imaging and demyelinating diseases: new aspects of an old advanced sequence. AJR Am J Roentgenol. 2014;202(1):W34-42. doi: 10.2214/AJR.13.11400. [PubMed: 24370163].

13. Lo CP, Kao HW, Chen SY, Chu CM, Hsu CC, Chen YC, et al. Comparison of diffusion-weighted imaging and contrast-enhanced T1-weighted imaging on a single baseline MRI for demonstrating dissemination in time in multiple sclerosis. BMC Neurol. 2014;14:100. doi:10.1186/14712377-14-100. [PubMed: 24885357].

14. Polman $\mathrm{CH}$, Reingold SC, Banwell B, Clanet M, Cohen JA, Filippi $M$, et al. Diagnostic criteria for multiple sclerosis: 2010 revisions to the McDonald criteria. Ann Neurol. 2011;69(2):292-302. doi: 10.1002/ana.22366. [PubMed: 21387374].

15. Jelescu IO, Leppert IR, Narayanan S, Araujo D, Arnold DL, Pike GB. Dualtemporal resolution dynamic contrast-enhanced MRI protocol for blood-brain barrier permeability measurement in enhancing multiple sclerosis lesions. J Magn Reson Imaging. 2011;33(6):1291-300. doi: 10.1002/jmri.22565. [PubMed: 21590997]. 
16. Huisman TA, Sorensen AG, Hergan K, Gonzalez RG, Schaefer PW. Diffusion-weighted imaging for the evaluation of diffuse axonal injury in closed head injury. J Comput Assist Tomogr. 2003;27(1):5-11. [PubMed: 12544235].

17. Rosso C, Remy P, Creange A, Brugieres P, Cesaro P, Hosseini H. Diffusion-weighted MR imaging characteristics of an acute strokelike form of multiple sclerosis. AJNR Am J Neuroradiol. 2006;27(5):1006-8. [PubMed: 16687533].

18. Bugnicourt JM, Garcia PY, Monet P, Bonnaire B, Al Khedr A, Godefroy O. Teaching NeuroImages: marked reduced apparent diffusion coefficient in acute multiple sclerosis lesion. Neurology. 2010;74(20):eee87. doi: 10.1212/WNL.0b013e3181dfo9f7. [PubMed: 20479354].

19. Eisele P, Szabo K, Griebe M, Rossmanith C, Forster A, Hennerici M, et al. Reduced diffusion in a subset of acute MS lesions: a serial multiparametric MRI study. AJNR Am J Neuroradiol. 2012;33(7):1369-73. doi: 10.3174/ajnr.A2975. [PubMed: 22576893].

20. Pagani E, Bammer R, Horsfield MA, Rovaris M, Gass A, Ciccarelli O, et al. Diffusion MR imaging in multiple sclerosis: technical aspects and challenges. AJNR Am J Neuroradiol. 2007;28(3):411-20. [PubMed: 17353305].

21. Iwasawa T, Matoba H, Ogi A, Kurihara H, Saito K, Yoshida T, et al.
Diffusion-weighted imaging of the human optic nerve: a new approach to evaluate optic neuritis in multiple sclerosis. Magn Reson Med. 1997;38(3):484-91. [PubMed: 9339450].

22. Bhatia R, Garg A. Diffusion abnormality detects an acute demyelinating lesion due to probable multiple sclerosis. Indian J Radiol Imaging. 2007;17(1):20

23. Balasubramanya KS, Kovoor JM, Jayakumar PN, Ravishankar S, Kamble RB, Panicker J, et al. Diffusion-weighted imaging and proton MR spectroscopy in the characterization of acute disseminated encephalomyelitis. Neuroradiology. 2007;49(2):177-83. doi: 10.1007/s00234-006-0164-2. [PubMed: 17131116].

24. Balashov KE, Aung LL, Dhib-Jalbut S, Keller IA. Acute multiple sclerosis lesion: conversion of restricted diffusion due to vasogenic edema. $J$ Neuroimaging. 2011;21(2):202-4. doi: 10.1111/j.1552-6569.2009.00443.x. [PubMed: 19888931].

25. Balashov KE, Lindzen E. Acute demyelinating lesions with restricted diffusion in multiple sclerosis. Mult Scler. 2012;18(12):1745-53. doi 10.1177/1352458512445407. [PubMed: 22523157].

26. Saharian M, Shakaouri Rad A, Motamedi M, Pakdaman H, Radue E. Magnetic resonance imaging abnormalities in multiple sclerosis: A review. Iran J Radiol. 2007;4(4):231-9. 